\section{Zusammenfassung}

Es wird eine Anordnung beschrieben, die einen Vergleich der Interferenzerscheinung bei kohärenter und inkohärenter Lage der Röntgen-Strahlquelle in bezug auf den Kristall gestattet. Die Tiefenlage der Quelle unter der Strahlaustrittsfläche kann verändert werden. Die Auswertung der (220)-Interferenzen an nahezu idealen Ge (ca. 2000 Versetzungen $/ \mathrm{cm}^{2}$ ) ergab zwischen Interferenzen bei kohärenter und inkohärenter Quellenlage folgende Unterschiede:

a) Bei kohärenter Quellenlage tritt bei zunehmender Tiefenlage der Quelle ein Umschlag der von Kossel beschriebenen Feinstruktur (HelldunkelStruktur) der Interferenzlinie ein.

b) Bei inkohärenter Quellenlage bleibt ein solcher Umschlag aus. Die Feinstruktur ist derjenigen bei kohärenter Quellenlage und geringer Quellentiefe entgegengesetzt.

c) Die Intensität der Interferenzen ist bei inkohärenter Quellenlage und größerer Kristalldicke bedeutend größer als bei kohärenter Quellenlage in der dieser Kristalldicke entsprechenden Tiefe.

Durch Einführung der Absorption in die v. Lauesche Theorie der Gitterquelleninterferenzen war ein Vergleich der experimentellen Ergebnisse mit den theoretisch zu erwartenden Interferenzkurven möglich. Es zeigte sich eine Übereinstimmung des Verhältnisses der experimentell bestimmten integralen Intensitäten bei kohärenter und inkohärenter Quellenlage mit dem theoretisch berechneten Verhältnis innerhalb der Fehlergrenzen.

Herrn Prof. Dr. W. Messerschmidt danken wir für das dieser Arbeit entgegengebrachte fördernde Interesse.

\title{
Zur Gittertheorie der piezoelektrischen und elastischen Eigenschaften von Kristallen mit Zinkblendestruktur unter Berüdksidhtigung der elektronischen Polarisation
}

II. Darstellung der piezoelektrischen Konstante $e_{\mathbf{1 4}}$ durch mikroskopische Parameter für das Modell einer reinen Feldpolarisation und für das Hüllen-Modell

\section{Von Ludwig Merten}

Aus der OSRAM-Studiengesellschaft, Augsburg

(Z. Naturforschg. 17 a, 216-227 [1962] ; eingegangen am 24. Dezember 1961)

\begin{abstract}
Die im ersten Teil erhaltenen allgemeinen Beziehungen werden im folgenden spezialisiert für konkrete, die elektronische Polarisation beschreibende Modelle. Von den nach Teil I durch die elektronische Polarisation beeinflußten makroskopischen Konstanten wird hier die besonders interessierende piezoelektrische Konstante $e_{14}$ im einzelnen untersucht, insbesondere, weil anzunehmen ist, da 3 die nach früheren Arbeiten erhaltenen noch relativ großen Diskrepanzen gegenüber dem experimentellen Wert in der Hauptsache auf dem nicht oder nur ungenügend berücksichtigten Einfluß der elektronischen Polarisation beruhen.

Zunächst wird das einfache Modell zugrunde gelegt, daß die Polarisation der Ionen allein durch das mit den Gitterschwingungen verknüpfte makroskopische elektrische Feld verursacht wird. Die sich für $\mathrm{ZnS}$ ergebende piezoelektrische Konstante $e_{14}$ ist aber hiernach um einen Faktor 7 bzw. 8 zu groß.

Nach dem Hüllen-Modell nach Dick, Overhauser und Cochrax werden die Ionen bei ihrer Auslenkung aber auch durch die nicht-Coulombschen Kräfte vor allem der nächsten Nachbarn polarisiert. Bei der numerischen Auswertung der für dieses Modell erhaltenen Gleichung zeigt sich, daß die Werte von $e_{14}$ für verschiedene Hüllenladungen des Anions in den meisten Fällen nur noch um ungefähr einen Faktor 2 bis 3 zu groß sind und auch der experimentelle Wert angenommen wird, und zwar für Hüllenladungen, die nach dem Vergleich mit anderen Untersuchungen als ganz vernünftig anzusehen sind. Insbesondere zeigt sich aber auch, daß $e_{\mathbf{1 4}}$ ganz empfindlich von einigen Parametern abhängt. Aus diesem Grunde und wegen der noch ungenauen Kenntnis der Eingangsdaten lassen sich allerdings über die Genauigkeit, mit der $e_{14}$ durch die erhaltenen Formeln dargestellt wird, erst endgültige Aussagen machen, wenn die Eingangsdaten genügend genau bekannt sind.
\end{abstract}

Wie bereits in Teil I * erwähnt wurde, ist anzunehmen, daß die in den bisherigen Arbeiten erhaltenen, noch relativ großen Diskrepanzen zwischen den Wer-

* L. Merten, Z. Naturforschg. 17 a, 174 [1962]. ten von $e_{14}$, die aus den Modellvorstellungen berechnet wurden, und dem experimentellen Wert in der Hauptsache darauf beruhen, daß der Einfluß der elektronischen Polarisation nicht oder nur ungenügend im Modell berücksichtigt wurde. 
Im folgenden Abschnitt II sollen daher die zwei folgenden oft betrachteten und als weitgehend gültig angesehenen Modelle zugrunde gelegt und speziell $e_{14}$ im Rahmen dieser Modelle berechnet werden. Zunächst wird $e_{14}$ dargestellt für das Modell einer reinen Feldpolarisation, bei dem also angenommen wird, daß die elektronische Polarisation allein eine Wirkung des (mit den Gitterschwingungen verknüpften) elektrischen Feldes ist. Es wird sich aber zeigen, daß die für $\mathrm{ZnS}$ erhaltenen Werte (im Absolutbetrag) viel zu groß sind. Dagegen ergeben sich wesentlich bessere Werte für das zweite Modell, nämlich das Hüllen-Modell, bei dem auf Grund der weiter unten genauer geschilderten Modellvorstellungen die Polarisation auch durch die nicht-Coulombschen Kräfte vor allem der nächsten Nachbarn wesentlich mitbedingt wird. Die Diskussion der Ergebnisse im einzelnen erfolgt im folgenden $\mathrm{Ab}$ schnitt.

\section{Berechnung der piezoelektrischen Konstante aus Modellvorstellungen}

\section{a) Modell einer reinen Feldpolarisation ${ }^{1}$}

Es sei also, wie oben bereits erwähnt, angenommen, daß die Polarisation allein durch das mit den Gitterschwingungen verknüpfte elektrische Feld verursacht wird und daß die von den Deformations. dipolen auf das betrachtete ausgelenkte Atom ausgeübten (Zusatz-) Kräfte rein elektrostatischer Natur sind.

Zwischen den induzierten Dipolmomenten $\boldsymbol{m}(k)$ und dem erregenden (effektiven) Felde $\boldsymbol{E}_{\text {eff }}$ besteht in erster Ordnung, und diese wird allein benötigt, ein linearer Zusammenhang, wobei der wegen der kubischen Symmetrie skalare Faktor bekanntlich als Polarisierbarkeit bezeichnet wird:

$$
\begin{aligned}
& \boldsymbol{m}^{(1)}(1)=\alpha_{1} \boldsymbol{E}_{\text {eff }}^{(1)}, \\
& \boldsymbol{m}^{(1)}(2)=\alpha_{2} \boldsymbol{E}_{\text {eff }}^{(1)} .
\end{aligned}
$$

Die Gln. (II, 1) stellen eine spezialisierte Form der

${ }^{1}$ Für optische Gitterschwingungen findet man allgemeine Rechnungen zu diesem Modell in Anm. 2, S. 272 ff. Für akustische Schwingungen sind jedoch explizite Rechnungen dort nicht durchgeführt.

2 M. Born u. K. Huang, Dynamical Theory of Crystal Lattices, Oxford, Clarendon Press 1954.

3 In nullter Ordnung würde sich das Lonentz-Feld

$$
\begin{aligned}
\boldsymbol{E}_{\mathrm{eff}}^{(0)} & =\boldsymbol{E}^{(0)}+Q_{o}\left(\boldsymbol{P}^{(0)}(1)+\boldsymbol{P}^{(0)}(2)\right) \\
& =\boldsymbol{E}^{(0)}+\frac{4 \pi}{3 v_{a}}\left(\boldsymbol{P}^{(0)}(\mathbf{1})+\boldsymbol{P}^{(0)}(2)\right)
\end{aligned}
$$

allgemeinen Gl. (I, 4) dar. Um mit ihr zu vergleichen, hat man noch $\boldsymbol{E}_{\text {eff }}^{(1)}$ als Funktion von $\boldsymbol{u}^{(0)}$, $\boldsymbol{u}^{(1)}(k), \boldsymbol{m}^{(1)}(k)$ und $\boldsymbol{E}^{(1)}$ darzustellen und einzusetzen. Dieser Zusammenhang wird (bis auf die von der speziellen Verbindung abhängige Gitterkonstante $a$ und die effektive Ionenladung $e$ ) durch die Kristallstruktur vollkommen festgelegt und läßt sich nach den von Bors und Huang ${ }^{2}$ beschriebenen Methoden berechnen [vgl. Gl. $(30,30))$, S. 254, bzw. Gl. $(35,8)$, S. 273] :

$$
\boldsymbol{E}_{\text {eff }}=\boldsymbol{E}+\sum_{k^{\prime}=1,2} Q\left(k k^{\prime}\right) \cdot \boldsymbol{P}\left(k^{\prime}\right) .
$$

Dabei ist $\boldsymbol{P}(k)$ das Gesamtdipolmoment:

$$
\boldsymbol{P}(k)=\boldsymbol{p}(k)+\boldsymbol{m}(k),
$$

mit $\boldsymbol{p}(k) \equiv e_{k} \boldsymbol{u}(k)$. Spezialisiert auf das Zinkblendegitter und die 1. Ordnung ${ }^{3}$ folgt hieraus (die Gleichungen und damit die effektiven Felder sind für $k=1$ und $k=2$ identisch) :

$$
\begin{aligned}
\boldsymbol{E}_{\mathrm{eff}}^{(1)}= & {\left[\boldsymbol{E}^{(1)}-e Q^{(1)}(12) \cdot \boldsymbol{u}^{(0)}-e Q_{0} \boldsymbol{u}^{(1)}\right] } \\
& +Q_{0}\left[\boldsymbol{m}^{(1)}(1)+\boldsymbol{m}^{(1)}(2)\right] .
\end{aligned}
$$

Dabei ist

$Q_{0} I \equiv Q^{(0)}(11)=Q^{(0)}(12)=\left[4 /\left(3 v_{a}\right)\right] I,(\mathrm{II}, 3 \mathrm{a})$ und $Q^{(1)}(12)$ hat die Gestalt:

$$
Q^{(1)}(12)=i a\left(p_{0} / v_{a}\right) q^{(1)} .
$$

Der Wert von $p_{0}$ ist durch die Kristallstruktur gegeben und wurde von verschiedenen Autoren unabhängig berechnet. Die Werte sind in Tab. 1 zusammengestellt. Außerdem sind in Tab. 1 aufge-

\begin{tabular}{|c|c|c|c|}
\hline & Verf. $^{4}$ & BLACKMAN $^{5}$ & TOLPYGO $^{6}$ \\
\hline$p_{0}$ & 2,51 & 2,51 & 2,514 \\
$c_{11}^{\mathrm{c}} / \frac{e^{2}}{a v_{a}}$ & 0,25 & 0,251 & 0,2475 \\
$c_{12}^{c} / \frac{e^{2}}{a v_{a}}$ & $-2,65$ & $-2,648$ & $-2,646$ \\
\hline
\end{tabular}

Tab. 1. Aus der Gittertheorie berechnete Werte für $p_{0}, c_{11}^{c}$ und $c_{12}^{\mathrm{c}}$.

ergeben, das aber für akustische Schwingungen wegen

$$
\begin{gathered}
\boldsymbol{m}^{(0)}(1)=\boldsymbol{m}^{(0)}(2)=\boldsymbol{E}^{(0)}=0 \\
\boldsymbol{p}^{(0)}(1)=e \boldsymbol{u}^{(0)}=-\boldsymbol{p}^{(0)}(2)
\end{gathered}
$$

verschwindet. Dagegen spielt es bei den optischen Schwingungen eine wesentliche Rolle.

${ }^{4}$ L. Merten, Diplomarbeit, Münster 1957, S. 76/77, Gln. (14) u. (15), u. Anm. ${ }^{8}$, S. 1075, Gl. (VII, 14) u. (VII, 16)

5 M. Blackman, Phil. Mag. 3, 831 [1958], speziell S. 833, Gl. (3).

${ }^{6}$ Siehe Anm. ${ }^{16}$ in Teil I, S. 7, Gl. (16) u. S. 11, Gl. (29). 
führt die aus der Gittertheorie berechneten Beiträge $c_{11}^{c}$ und $c_{12}^{c}$ der Coulomв-Kräfte zu den elastischen Konstanten, die zur späteren numerischen Berechnung der Kopplungsparameter $B$ und $C$ benutzt sind [vgl. (II, 15a)].

Um $\boldsymbol{m}^{(1)}(1)$ und $\boldsymbol{m}^{(1)}(2)$ zu berechnen, kürzen wir die erste Klammer in (II, 3) durch $\boldsymbol{K}^{(1)} \mathrm{ab}$. setzen (II, 3) in (II, 1) ein und fassen die Dipolmomente auf der linken Seite zusammen:

$$
\begin{aligned}
& \left(1-\alpha_{1} Q_{0}\right) \boldsymbol{m}^{(1)}(1)-\alpha_{1} Q_{0} \boldsymbol{m}^{(1)}(2)=\alpha_{1} \boldsymbol{K}^{(1)}, \\
& -\alpha_{2} Q_{0} \boldsymbol{m}^{(1)}(1)+\left(1-\alpha_{2} Q_{0}\right) \boldsymbol{m}^{(1)}(2)=\alpha_{2} \mathbf{K}^{(1)} .
\end{aligned}
$$

Nach Multiplikation beider Seiten mit der zur Koeffizientenmatrix reziproken Matrix

$$
\frac{1}{1-\left(\alpha_{1}+\alpha_{2}\right) Q_{0}}\left(\begin{array}{cc}
1-\alpha_{2} Q_{0} & \alpha_{1} Q_{v} \\
\alpha_{2} Q_{0} & 1-\alpha_{1} Q_{0}
\end{array}\right)
$$

folgt

$$
\begin{aligned}
& \boldsymbol{m}^{(1)}(1)=\frac{\alpha_{1}}{1-\alpha Q_{0}} \boldsymbol{K}^{(1)}, \\
& \boldsymbol{m}^{(1)}(2)=\frac{\alpha_{2}}{1-\alpha Q_{0}} \boldsymbol{K}^{(1)} .
\end{aligned}
$$

$\alpha$ ist dabei die Summe der Polarisierbarkeiten beider Atome: $\alpha=\alpha_{1}+\alpha_{2}$. - Indem man $\boldsymbol{K}^{(1)}$ aus (II, 3) einsetzt, erhält (II, 5) die Gestalt von (I, 4). Der Vergleich ergibt

$$
\begin{array}{cl}
K_{1}=\alpha_{1} / D, & L_{1}=-\left(\alpha_{1} / D\right) e Q_{0}, \\
K_{2}=\alpha_{2} / D, & L_{2}=-\left(\alpha_{2} / D\right) e Q_{0} \\
\text { mit } & D=1-\alpha Q_{0} .
\end{array}
$$

$\left[L_{1}{ }^{(1)}\right.$ und $L_{2}{ }^{(1)}$ benötigen wir hier nicht explizit, da wir hier zur Berechnung von $e_{14} \mathrm{Gl}$. (I, 13) benutzen, in die sie nicht eingehen.]

Die weiteren Größen erhält man aus Gl. (1) bzw. Gl. (I, lb) in Teil I. Dabei sei ${ }^{7} C\left(k k^{\prime}\right)$ wie in ${ }^{2,8}$ (vgl. auch Anm. ${ }^{5}$ in Teil I) aufgespalten in einen Term $C_{\mathrm{N}}\left(k k^{\prime}\right)$ für die Kräfte kurzer Reichweite und einen $B\left(k k^{\prime}\right)=-e_{k} e_{k^{\prime}} Q\left(k k^{\prime}\right)$ für die CoulombKräfte ${ }^{9}$

$$
\begin{aligned}
C^{(1)}\left(k k^{\prime}\right) & =C_{\mathrm{N}}^{(1)}\left(k k^{\prime}\right)+B^{(1)}\left(k k^{\prime}\right) \\
& =C_{\mathbf{N}}^{(1)}\left(k k^{\prime}\right)-e_{k} e_{k^{\prime}} Q^{(1)}\left(k k^{\prime}\right) .
\end{aligned}
$$

Für die Größen erster Ordnung erhält man

und

$$
C_{\mathrm{N}}^{(1)}(12)=-C_{\mathrm{N}}^{(1)}(21)=-i a r q^{(1)}
$$

$$
B^{(1)}(12)=-B^{(1)}(21)=e^{2} Q^{(1)}(12)=i a p q^{(1)},
$$

\footnotetext{
7 Diese Summe ist mit der in Anm. ${ }^{6}$ in Teil I als $C_{G}\left(k k^{\prime}\right)$ bezeichneten identisch.

${ }^{8}$ Siehe Anm. ${ }^{6}$ in Teil I.
}

wobei $r$ nach $^{8}$ [vgl. Gl. (IV, 10a), S. 672] durch die Kopplungsparameter z. B. erster bis dritter Nachbaratome explizit wie folgt dargestellt wird

$$
r \equiv-a(c+j+k-3 n)
$$

und $p$ mit $p_{0}[\mathrm{Gl}$. (II, $\left.3 \mathrm{~b})\right]$ in folgendem Zusammenhang steht:

$$
p=p_{0}\left(e^{2} / v_{a}\right) .
$$

Man erhält somit:

$C^{(1)}=C^{(1)}(12)=i c^{1} q^{(1)}=-i a(r-p) q^{(1)}$.

Um die von den Deformationsdipolen ausgeübten Kräfte zu erhalten, hat man nur - wegen der Voraussetzung, daß die Kräfte rein elektrostatisch sind in dem zum zweiten Glied in (II, 7) gehörigen Term in Gl. (1) aus Teil I, nämlich

$-e_{k} e_{k^{\prime}} Q\left(k k^{\prime}\right) \cdot \boldsymbol{u}\left(k^{\prime}\right)=-e_{k} Q\left(k k^{\prime}\right) \cdot \boldsymbol{p}\left(k^{\prime}\right),(\mathrm{II}, 9)$

die Auslenkungsdipolmomente $\boldsymbol{p}\left(k^{\prime}\right)=e_{k^{\prime}} \boldsymbol{u}\left(k^{\prime}\right)$ durch die Deformationsdipolmomente $\boldsymbol{m}\left(k^{\prime}\right)$ zu ersetzen. Somit ergibt der Vergleich mit Gl. (1) in Teil I:

$$
\begin{aligned}
& M_{1}^{(1)}=M^{(1)}(21)=e Q^{(1)}(21)=-i \frac{a p}{e} q^{(1)}, \\
& M_{2}^{(1)}=M^{(1)}(12)=-e Q^{(1)}(12)=-i \frac{a p}{e} q^{(1)}, \\
& \text { d. h. } \quad m_{1}{ }^{1}=m_{2}{ }^{1}=-a p / e .
\end{aligned}
$$

In der nullten Ordnung ergibt sich

$$
\begin{aligned}
C & =C_{X 11}^{(0)}(12)+e^{2} Q_{11}^{(0)}(12)=-R_{0}+e^{2} Q_{0}, \\
M_{1} & =M_{2}=M^{(0)}(11)=M^{(0)}(12)=-e Q_{0} .
\end{aligned}
$$

(Der Index 11 bezeichnet dabei eines der drei übereinstimmenden Diagonalelemente der Glieder nullter Ordnung.) Mit der Abkürzung $R_{\mathbf{0}}=-4(b+h+21)$ sind dabei wieder in ${ }^{8}$ [vgl. z. B. Gl. (IV, 9c)] definierte Kopplungsparameter zusammengefaßt.

Nach Einsetzen von (II, 6) und (II, 11) ergibt sich für $U$ [Gl. $(\mathrm{I}, 5 \mathrm{~b})$ in Teil I] mit

$$
-\left(M_{1} K_{1}+M_{2} K_{2}-e\right)=e\left(\frac{a Q_{0}}{D}+1\right)=\frac{e}{D}
$$

als Zähler und

$$
\begin{aligned}
C+M_{1} L_{1}+M_{2} L_{2} & =-R_{0}+e^{2} Q_{0}+\alpha e^{2} Q_{0}{ }^{2} / D \\
& =-\left(R_{0} D-e^{2} Q_{0}\right) / D
\end{aligned}
$$

als Nenner:

$$
U=-e /\left(R_{0} D-e^{2} Q_{0}\right),
$$

${ }^{9}$ Im Gegensatz zu ${ }^{8}$ ist im folgenden die Darstellung mit Hilfe $\operatorname{der} Q\left(k k^{\prime}\right)$ [statt $\operatorname{der} B\left(k k^{\prime}\right)$ ] bequemer, die sich an $^{2}$ [vgl. z. B. Gl. (30, 31), S. 254] anschließt. 
also mit (II, 8)

$$
c^{1} U=a e(r-p) /\left(R_{0} D-e^{2} Q_{0}\right) .
$$

Damit folgt nach (I, 6d)

$$
V_{1(2)}=\frac{a_{1(2)}}{D}\left(-e Q_{0} U+1\right)=\frac{a_{1(2)} R_{0}}{R_{0} D-e^{2} Q_{0}}
$$

und mit (II, 10a) :

$$
m_{1}{ }^{1} V_{1}+m_{1}{ }^{2} V_{2}=-a e \frac{a R_{0}\left(p / e^{2}\right)}{R_{0} D-e^{2} Q_{0}},
$$

also nach $(I, 13)$ als Endergebnis:

$$
e_{14}=-\frac{4 e}{a^{2}} \frac{a R_{0}\left(p / e^{2}\right)-(r-p)}{R_{0} D-e^{2} Q_{0}}
$$

Berücksichtigen wir schließlich von den Kräften kurzer Reichweite nur diejenigen zwischen nächsten Nachbarn, d. h. setzen ${ }^{10}$

$$
R_{0}=-4 b=4 B, \quad r=-c=C,
$$

so vereinfacht sich obige Gleichung zu

$$
e_{14}=-\frac{4 e}{a^{2}} \frac{4 a B\left(p / e^{2}\right)-(C-p)}{4 B D-e^{2} Q_{0}},
$$

und alle Größen in ihr sind bestimmbar: $B$ und $C$ ergeben sich wie in Anm. ${ }^{8}$, S. 1075, aus den elastischen Konstanten $c_{11}$ und $c_{12}$, nämlich nach

$$
\begin{aligned}
& B=a\left(c_{11}-c_{11}^{c}\right), \\
& C=\frac{1}{2} a\left(c_{11}+c_{12}-c_{11}^{c}-c_{12}^{c}\right),
\end{aligned}
$$

$e$ aus der Formel von Szigeti (vgl. ${ }^{2}$, S. 112). a läßt sich schließlich aus der Formel von LonentzLORENz bestimmen:

$$
\alpha=\frac{3 v_{\mathrm{a}}}{4} \frac{\varepsilon_{\infty}-1}{\varepsilon_{\infty}+2} .
$$

Die für $\mathrm{ZnS}$ benötigten Daten sind in Tab. 2 zusammengestellt.

Mit den in Tab. 2 angegebenen Werten ergibt sich

$$
e_{14}=-\begin{array}{rr}
31,0 \cdot 10^{4} & \text { (l. c. } \left.{ }^{11}\right) \\
35,0 \cdot 10^{4} & \text { (l. c. } \left.{ }^{12}\right)
\end{array}
$$

$$
\text { [el.stat. cgs } / \mathrm{cm}^{2}=\mathrm{cm}^{-1 / 2} \mathrm{~g}^{1 / 2} \mathrm{sec}^{-1} \text { ]. }
$$

Vergleicht man die berechneten Werte mit dem experimentellen Wert ${ }^{13} e_{14}=-4,2 \cdot 10^{4}$, so sind jene also um den Faktor 7,4 bzw. 8,3 zu groß. Diese Diskrepanz ist jedoch nicht mehr so verwunderlich, wenn man sich vergegenwärtigt, wie empfindlich das Ergebnis insbesondere vom benutzten Modell für die Deformationspolarisation abhängt (im Zähler wird die Differenz zweier Zahlen von gleicher Größenordnung gebildet). Vernachlässigt man die Polarisation z. B. gänzlich, d. h. setzt $\alpha=0$, so schlägt das Vorzeichen des Zählers $\left(=-41,70 \cdot 10^{3} \mathrm{dyn} / \mathrm{cm}\right)$ sogar um, so daß auch $e_{14}$ selbst das Vorzeichen wechselt $\left(\text { Nenner }=205,7 \cdot 10^{3} \mathrm{dyn} / \mathrm{cm}\right)^{14}$ :

$$
e_{14}=12,8 \cdot 10^{4} \text { el.stat.cgs } / \mathrm{cm}^{2} \text {. }
$$

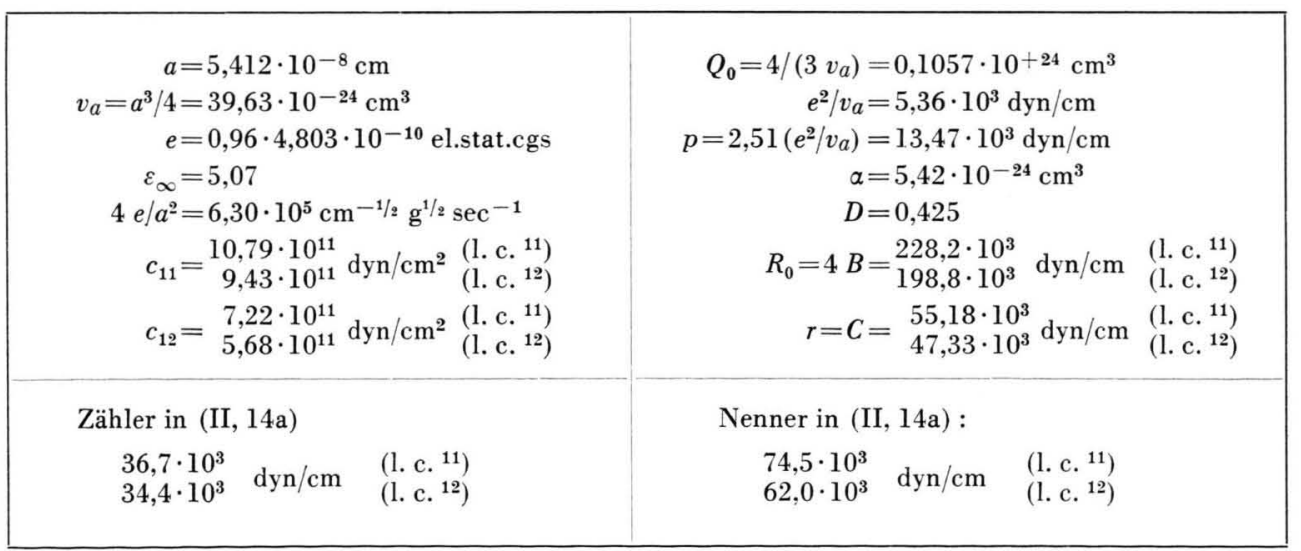

Tab. 2. Numerische Werte für $\mathrm{ZnS}$ der in $e_{14}$ eingehenden Größen.

10 Vielfach werden die Kopplungsparameter auch durch kleine griechische Buchstaben bezeichnet, und zwar besteht zu den hier und in ${ }^{8}$ benutzten Kopplungsparametern $B$ und $C$ die Zuordnung: $\alpha_{1}=B, \beta_{1}=C$. Eine Einführung dieser griechischen Buchstaben würde aber gerade in Formel (II, 14a) leicht zu Verwechslungen mit den Polarisierbarkeiten $\left(\alpha=\alpha_{1}+\alpha_{2}\right)$ führen.
11 Elastische Konstanten nach S. Bhagavantam u. D. Suryanarayana, Proc. Ind. Acad. Sci. 42, 304 [1944].

12 Elastische Konstanten nach W. Vorgt, Nachr. Ges. Wiss. Göttingen, Math.-phys. Kl. 1918, S. 424-456.

13 W. CAdy, Piezoelectricity, McGraw.Hill Book Company, New York 1946, S. 229.

14 Die Zahlenwerte gelten bei Benutzung der elastischen Konstanten nach Anm. ${ }^{11}$. 
Da die Kopplungsparameter zweiter Nachbarn gar nicht eingehen ${ }^{15}$ und von dritten Nachbarn kein wesentlicher Einfluß mehr zu erwarten ist, ist anzunehmen, daß die wesentlichen Diskrepanzen nur darauf beruhen, daß die benutzten Modellvorstellungen hinsichtlich der Deformationspolarisation nur ungenügend bei den realen Kristallen zutreffen. Im folgenden Abschnitt b) sollen deshalb modernere Vorstellungen über die Ursache der Polarisation im Modell berücksichtigt und speziell bezüglich ihres Einflusses auf den Piezoeffekt untersucht werden.

\section{b) Modell von Dick, Overhauser und Cochran (Hüllen-Modell)}

In der Formel (II, 1) wurde vorausgesetzt, daß die Polarisation der Ionen allein durch das elektrische Feld verursacht wird. Ein Ion wird aber bei seiner Auslenkung auch durch die (nicht-Coulombschen) Bindungskräfte zumindest mit den nächsten Nachbarn deformiert, und zwar so, daß sich die Ladungsschwerpunkte von Hülle und Rumpf (= Kern + innere Elektronen) gegeneinander verschieben werden, wodurch ein zusätzliches (Deformations-) Dipolmoment entsteht. Wie in der Einleitung bereits bemerkt wurde, erfolgt nach dem Modell von Dick und Overhauser ${ }^{16}$ (Hüllen-[shell-] Modell) die Verschiebung der Schwerpunkte in erster Näherung da- durch, daß die Hülle der Valenzelektronen in sich starr gegenüber dem Rumpf verschoben wird. Die Hülle ist dabei durch eine Kraftkonstante (Federkonstante) mechanisch mit dem Rumpf gekoppelt. Da dieses Modell von Cochran erfolgreich zur Deutung der Gitterschwingungen sowohl bei den fast rein ionogenen Alkalihalogeniden (genauere Rechnungen wurden für $\mathrm{NaJ}^{17}$ durchgeführt) als auch bei den rein homöopolaren Kristallen wie Germanium ${ }^{18}$ angewandt wurde, ist anzunehmen, daß es auch für Kristalle mit Zinkblendestruktur angenähert zutrifft, die ja bezüglich ihres Bindungscharakters zwischen diesen beiden Verbindungstypen stehen. Insbesondere wird man daher auch erwarten, daß es zu einer weitgehend befriedigenden Darstellung der piezoelektrischen Konstante führt.

In diesem Abschnitt soll daher die piezoelektrische Konstante in diesem Modell dargestellt und die erhaltenen numerischen Werte mit dem experimentellen verglichen werden. Einer etwaigen teilweise homöopolaren Bindung der Kristalle mit Zinkblendestruktur tragen wir dadurch Rechnung, daß wir Abweichungen von der Kugelsymmetrie der Hülle zulassen, indem wir allgemeine, nur die Kristallsymmetrie berücksichtigende Kopplungsparameter benutzen und außerdem mit „effektiven“ Ladungen der Ionen rechnen.

Die Schwingungsgleichungen in dem Modell von Dick, Overhauser und Cochran lauten jetzt ${ }^{19}$ : $m_{k} \omega^{2} \boldsymbol{u}(k)=\sum_{k^{\prime}}\left[C_{\mathrm{N}}\left(k k^{\prime}\right)-e_{k} e_{k^{\prime}} Q\left(k k^{\prime}\right)\right] \cdot \boldsymbol{u}\left(k^{\prime}\right)+\sum_{k^{\prime}}\left[T\left(k k^{\prime}\right)-e_{k} y_{k^{\prime}} Q\left(k k^{\prime}\right)\right] \cdot\left(\boldsymbol{u}_{\mathrm{H}}\left(k^{\prime}\right)-\boldsymbol{u}\left(k^{\prime}\right)\right)-e_{k} \boldsymbol{E}$ oder, wenn man die Coulomb-Terme zu einem effektiven Feld zusammenfaßt:

$$
\left(k, k^{\prime}=1,2\right), \quad(\mathrm{II}, 16)
$$

$$
m_{k} \omega^{2} \boldsymbol{u}(k)=\sum_{k^{\prime}=1,2} C_{\mathrm{N}}\left(k k^{\prime}\right) \cdot \boldsymbol{u}\left(k^{\prime}\right)+\sum_{k^{\prime}=1,2} T\left(k k^{\prime}\right) \cdot\left(\boldsymbol{u}_{\mathrm{H}}\left(k^{\prime}\right)-\boldsymbol{u}\left(k^{\prime}\right)\right)-e_{k} \boldsymbol{E}_{\mathrm{eff}},
$$

wobei

$$
\boldsymbol{E}_{\mathrm{eff}}=\boldsymbol{E}+\sum_{k^{\prime}=1,2} Q\left(k k^{\prime}\right) \cdot\left[e_{k^{\prime}} \boldsymbol{u}\left(k^{\prime}\right)+y_{k^{\prime}}\left(\boldsymbol{u}_{\mathrm{H}}\left(k^{\prime}\right)-\boldsymbol{u}\left(k^{\prime}\right)\right)\right] .
$$

Die Tensoren (Kopplungsdyaden) wurden dabei wieder aufgespalten in diejenigen für nicht-Coulombsche (kurze Reichweite) und diejenigen für Coulombsche Kräfte.

Die Annahme des Hüllenmodells bedeutet dabei, daß die Größen

$$
C_{\mathrm{N}}\left(k k^{\prime}\right)=D\left(k k^{\prime}\right)+S\left(k k^{\prime}\right)+2 F\left(k k^{\prime}\right)(\mathrm{II}, 16 \mathrm{c})
$$

15 Ihr Einfluß kann sich höchstens indirekt bemerkbar machen, da sie in den Gln. für $c_{11}$ und $c_{12}$ auftreten, die zur Bestimmung von $B$ und $C$ benutzt sind.

16 Siehe Anm. ${ }^{14}$ in Teil I.

17 Siehe Anm. ${ }^{15 b}$ in Teil I.

18 Siehe Anm. ${ }^{15}$ a in Teil I.

19 In der Bezeichnung schließen wir uns weitgehend ${ }^{2}$ bzw. ${ }^{17}$

$$
\text { und } T\left(k k^{\prime}\right)=S\left(k k^{\prime}\right)+F\left(k k^{\prime}\right)
$$

eine Summe von Termen darstellen, die folgenden Kräften kurzer Reichweite zugeordnet sind:

an, beschränken uns aber nicht wie in ${ }^{17}$ von vornherein auf spezielle Richtungen. Die Zusammenfassung sämtlicher Coulomb-Terme in $\boldsymbol{E}_{\text {eff }}$ in Gl. (II, 16) und Gl. (II, 17) und späteren Gleichungen bedeutet gegenüber entsprechenden Gleichungen in ${ }^{17}$ nur einen formalen Unterschied. Dies erleichtert aber die Übersicht und den Vergleich mit dem Modell a). 
$D\left(k k^{\prime}\right)$ : Rümpfe $k^{\prime}$ auf Rumpf $k$,

$S\left(k k^{\prime}\right)$ : Hüllen der Rümpfe $k^{\prime}$ auf Hülle des Rumpfes $k$,

$F\left(k k^{\prime}\right)$ : Rümpfe $k^{\prime}$ auf Hülle des Rumpfes $k$,

$F^{\prime}\left(k k^{\prime}\right)$ : Hüllen der Rümpfe $k^{\prime}$ auf Rumpf $k$ [ $\left.=F\left(k k^{\prime}\right)\right]$.

In (II, 16) wurde dabei wie in ${ }^{17} F=F^{\prime}$ gesetzt. $\mathrm{Ob}$ dies auch physikalisch gerechtfertigt ist, sei hier offen gelassen. Zumindest kann diese Gleichsetzung als eine Mittelwertbildung angesehen werden, und sie vereinfacht die Rechnung. Bei den späteren numerischen Auswertungen wird ohnehin vorausgesetzt, daß $F=F^{\prime}=0$.

$\boldsymbol{u}_{\mathrm{H}}(k)$ bedeutet die Verrückung des Schwerpunktes der Hülle des Rumpfes $k$, also $\boldsymbol{u}_{\mathrm{H}}(k)-\boldsymbol{u}(k)$ die relative Verschiebung der Ladungsschwerpunkte von Hülle und Rumpf. $y_{k}$ ist die Ladung der Hülle des Rumpfes $k$. Zur genaueren Diskussion der Grundgleichungen ${ }^{19}$ siehe insbesondere Cochran ${ }^{17}$. Die Verrückungen der Atome sind mit den Schwerpunktsverschiebungen zwischen Hülle und Rumpf wie folgt verknüpft:

$$
\begin{aligned}
& 0=\sum_{k^{\prime}} T\left(k k^{\prime}\right) \cdot \boldsymbol{u}\left(k^{\prime}\right) \\
& +\left[T(k k)+k_{k} I\right] \cdot\left(\boldsymbol{u}_{\mathrm{H}}(k)-\boldsymbol{u}(k)\right) \\
& +S\left(k k^{\prime}\right) \cdot\left(\boldsymbol{u}_{\mathrm{H}}\left(k^{\prime}\right)-\boldsymbol{u}\left(k^{\prime}\right)\right)-y_{k} \boldsymbol{E}_{\mathrm{eff}} \\
& \left(k, k^{\prime}=1,2\right) \text {. }
\end{aligned}
$$

$k_{1}, k_{2}$ bedeuten dabei die Kraftkonstanten, die die Hüllen mit ihren zugehörigen Rümpfen 1 bzw. 2 koppeln.

Um die Gleichungen auf die allgemeine Form der Gl. (1) und Gl. (I, 4) (Teil I) zu bringen, hat man noch die Deformationsdipolmomente einzuführen.

$$
\boldsymbol{m}(k)=y_{k}\left(\boldsymbol{u}_{\mathrm{H}}(k)-\boldsymbol{u}(k)\right) .
$$

Die Gln. (II, 16) lauten dann:

$$
\begin{aligned}
m_{1} \omega^{2} \boldsymbol{u}(1)= & {\left[C_{\mathrm{N}}(11)-e^{2} Q(11)\right] \cdot \boldsymbol{u}(1)(\mathrm{II}, 19 \mathrm{a}) } \\
& +\left[C_{\mathrm{N}}(12)+e^{2} Q(12)\right] \cdot \boldsymbol{u}(2) \\
& +\left[T(11) / y_{1}-e Q(11)\right] \cdot \boldsymbol{m}(1) \\
& +\left[T(12) / y_{2}-e Q(12)\right] \cdot \boldsymbol{m}(2)-e \boldsymbol{E}, \\
m_{2} \omega^{2} \boldsymbol{u}(2)= & {\left[C_{\mathrm{N}}(21)+e^{2} Q(21)\right] \cdot \boldsymbol{u}(1)(\mathrm{II}, 19 \mathrm{~b}) } \\
& +\left[C_{\mathrm{N}}(22)-e^{2} Q(22)\right] \cdot \boldsymbol{u}(2) \\
& +\left[T(21) / y_{1}+e Q(21)\right] \cdot \boldsymbol{m}(1) \\
& +\left[T(22) / y_{2}+e Q(22)\right] \cdot \boldsymbol{m}(2)+e \boldsymbol{E} .
\end{aligned}
$$

20 Die Tensoren $T^{(1)}(11)$ und $T^{(1)}(22)$ brauchen dabei nicht berücksichtigt werden, da sie in der betrachteten Näherung verschwinden (vgl. auch Anhang zu Teil I).
In den Gln. (II, 17) gehen wir zweckmäßig sofort zur ersten Ordnung über, die allein benötigt wird. Um diese Gleichungen auf die Form $(\mathrm{I}, 4)$ zu bringen, sind sie noch mit $\alpha_{1} / y_{1}$ bzw. $\alpha_{2} / y_{2}$ zu multiplizieren, wobei

$$
\alpha_{1} \equiv \frac{y_{1}^{2}}{T_{0}+k_{1}} \quad \text { bzw. } \quad \alpha_{2} \equiv \frac{y_{2}^{2}}{T_{0}+k_{2}}
$$

und

$$
\begin{aligned}
T_{0} I \equiv & T^{(0)}(11)=T^{(0)}(22) \\
& =-T^{(0)}(12)=-T^{(0)}(21),
\end{aligned}
$$

und $\boldsymbol{m}^{(1)}(1)$ bzw. $\boldsymbol{m}^{(1)}(2)$ nach links zu bringen ${ }^{20}$ :

$$
\begin{aligned}
\boldsymbol{m}^{(1)}(1)= & \alpha_{1} \boldsymbol{E}_{\mathrm{eff}}^{(1)}-\frac{\alpha_{1}}{y_{1}} T^{(1)}(12) \cdot \boldsymbol{u}^{(0)} \\
& +\frac{\alpha_{1}}{y_{1}} T^{(0)}(11) \cdot \boldsymbol{u}^{(1)} \\
& -\frac{\alpha_{1}}{y_{1} y_{2}} S^{(0)}(12) \cdot \boldsymbol{m}^{(1)}(2), \\
\boldsymbol{m}^{(1)}(2)= & \alpha_{2} \boldsymbol{E}_{\mathrm{eff}}^{(1)}-\frac{\alpha_{2}}{y_{2}} T^{(1)}(21) \cdot \boldsymbol{u}^{(0)} \\
& -\frac{\alpha_{2}}{y_{2}} T^{(0)}(22) \cdot \boldsymbol{u}^{(1)} \\
& -\frac{\alpha_{2}}{y_{1} y_{2}} S^{(0)}(21) \cdot \boldsymbol{m}^{(1)}(1) .
\end{aligned}
$$

Neben (II, 20a) führen wir dabei für später noch die Abkürzung ein :

$$
S_{0} I=-S^{(0)}(12)=-S^{(0)}(21) .
$$

$T^{(1)}$ (12) hat nach Gl. (I, 2) aus Teil I die Gestalt:

$$
T^{(1)}(12)=-T^{(1)}(21)=-i a t q^{(1)} .
$$

Die drei letzten Glieder in (II, 21), die offensichtlich den Einfluß der Kräfte kurzer Reichweite auf die Polarisation der Gitterbausteine beschreiben, treten jetzt gegenüber Gl. (II, 1) hinzu. - Als $\boldsymbol{E}_{\text {eff }}^{(1)}$ hat man die erste Ordnung von (II, 16b) einzusetzen, die mit Gl. (II, 3) identisch ist.

Für eine erste Näherung wird es sicherlich wie bei vielen Alkalihalogeniden erlaubt sein, nur die $\mathrm{P}_{0}$ larisierbarkeit des Anions (z. B. des S-Ions in $\mathrm{ZnS}$ ) zu berücksichtigen; die Polarisierbarkeit z. B. des freien $\mathrm{Zn}^{++}$-Ions ist nämlich nur ${ }^{21} \alpha_{1}=0,285 \cdot 10^{-24}$ $\mathrm{cm}^{-3}$, also ungefähr 19-mal so klein wie die Gesamtpolarisierbarkeit $\alpha$ (vgl. Tab. 2, S. 219). Wir dürfen also $\alpha=\alpha_{2}$ und $\boldsymbol{m}^{(1)}(1)=0$ setzen. Man muß hierbei allerdings bedenken, daß die Polarisierbarkeit $\alpha_{1}$ (und natürlich auch $\alpha_{2}$ ) sich durch den Ein-

21 Landolt-Börnstein, Springer-Verlag 1950, Bd. 1, Teil 1, S. 402 . 
bau in den Kristall, u. a. auch durch homöopolare Bindungsanteile, etwas ändern wird, aber sicherlich nicht so stark, daß eine Vernachlässigung von $\alpha_{1}$ gegenüber $\alpha_{2}$ nicht mehr berechtigt ist. Im Anhang ist zur Vollständigkeit auch der allgemeine Fall durchgerechnet, in dem die Dipolmomente beider Gitterbausteine berücksichtigt sind.

Da die Kräfte von zweiten (vierten usw.) Nachbarn in die Tensoren nullter und erster Ordnung ${ }^{22}$ und folglich auch nicht in $e_{14}$ eingehen, berücksichtigen wir außerdem von den Kräften kurzer Reichweite nur die von ersten Nachbarn (vernachlässigen also diejenigen von dritten und höheren Nachbarn) . Die Faktoren $C^{(n)}\left(k k^{\prime}\right)$ brauchen wir nicht erneut anzuführen, da sie natürlich dieselbe Gestalt wie in den bisher betrachteten Modellen besitzen. Wir haben also nur die zu den Dipolmomenten gehörigen Faktoren neu zu berechnen. Wegen der Voraussetzung $\boldsymbol{m}^{(1)}(1)=0$ benötigen wir nur die zweite der Gln. (II, 21), die nach Einsetzen von (II, 3) jetzt lautet:

$$
\boldsymbol{m}^{(1)}(2)=\frac{\alpha}{D}\left[\boldsymbol{K}^{(1)}-\frac{T^{(1)}(21)}{y_{2}} \cdot \boldsymbol{u}^{(0)}-\frac{T_{0}}{y_{2}} \boldsymbol{u}^{(1)}\right],
$$

wobei $\boldsymbol{K}^{(1)}$ wieder die erste eckige Klammer in (II, 3) bedeutet. Durch Vergleich mit Gl. (I, 4) liest man $a b$ :

$$
K_{2}=\frac{\alpha}{D}, \quad L_{2}=-\frac{\alpha}{D}\left[e Q_{0}+\frac{T_{0}}{y_{2}}\right]=-\frac{\alpha}{D} E,
$$

mit der Abkürzung $E \equiv e Q_{0}+T_{0} / y_{2}$.

Schließlich ergibt sich aus dem Vergleich von (II, 19a) mit (I, l'b) aus Teil I für die nullte Ordnung von $M(12)$ :

$$
M_{2} I=M^{(0)}(12)=-\left(T_{0} / y_{2}+e Q_{0}\right) I=-E I,
$$

und für die erste Ordnung [man vergleiche mit (I, 1c) und (I, 3) in Teil I] :

$$
M^{(1)}(12)=\frac{T^{(1)}(12)}{y_{2}}-e Q^{(1)}(12)=i m_{2}{ }^{1} q^{(1)},
$$

d. h. nach (II, 8b) und (II, 20c) :

$$
m_{2}{ }^{1}=-a\left(\frac{t}{y_{2}}+\frac{p}{e}\right) \text {. }
$$

Mit

$$
U_{\mathrm{Z}}=-M_{2} K_{2}+e=\frac{e}{D}\left(1+\frac{a T_{0}}{e y_{2}}\right)
$$

22 Von der im Anhang zu Teil I, S. 179, erwähnten möglichen Ausnahme, daß sie in einige der Tensoren erster Ordnung „schwach" eingehen könnten, sei dabei abgesehen. als Zähler und

$$
U_{\mathrm{N}}=C+M_{2} L_{2}=-\frac{\left(R_{0}-e^{2} Q_{0}\right) D-E^{2}}{D}=-\frac{U^{\prime}}{D}
$$

als Nenner von $U$ und Gl. (JI, 8e) erhält man also:

$$
c^{1} U=-e a^{(r-p)\left[1+\alpha T_{0} /\left(e y_{2}\right)\right]} U^{\prime} .
$$

Dabei wurde abgekürzt:

$$
\begin{aligned}
U^{\prime} & =D\left(R_{0}-e^{2} Q_{0}\right)-\alpha E^{2} \\
& =R_{0} D-e^{2} Q_{0}-\alpha \frac{T_{0}}{y_{2}}\left(2 e Q_{0}+\frac{T_{0}}{y_{2}}\right) .
\end{aligned}
$$

Weiter ergibt sich für $V_{2}$ [vgl. (I, 6d) ] :

$$
\begin{aligned}
V_{2} & =L_{2} U+K_{2}=\frac{\alpha}{D U_{\mathrm{N}}}\left[-U_{\mathrm{Z}} E+U_{\mathrm{N}}\right] \\
& =\frac{\alpha}{U^{\prime}}\left[R_{0}+\frac{e}{y_{2}} T_{0}\right]
\end{aligned}
$$

und mit Gl. (II, 24c)

$m_{2}{ }^{1} V_{2}=-e a \frac{a p R_{0}}{e^{2}}\left(1+\frac{t}{p} \frac{e}{y_{2}}\right)\left(1+\frac{T_{0}}{R_{0}} \frac{e}{y_{2}}\right) / U^{\prime}$.

Aus (II, 26) und (II, 28) folgt schließlich nach (I, 13) als Endergebnis

$$
\begin{aligned}
e_{14}=-\frac{4 e}{a^{2}}\left[\frac{\alpha p R_{0}}{e^{2}}\right. & \left(1-\frac{r}{p} \frac{e}{Y}\right)\left(1-\frac{e}{Y}\right) \\
& \left.-(r-p)\left(1-\frac{\alpha}{e} \frac{R_{0}}{Y}\right)\right] / U^{\prime} .
\end{aligned}
$$

In der Endformel (II, 29) wurde der absolute Wert der Hüllenladung $Y=\left|y_{2}\right|=-y_{2}$ der Atome 2 eingeführt, womit dann alle auftretenden Größen positive Werte annehmen. Außerdem wurde

$$
T_{0}=R_{0} \quad \text { und } \quad t=r
$$

gesetzt. Dies ergibt sich aus folgender Voraussetzung: Wir nehmen mit Cochran ${ }^{17}$ an, daß die (nichtCouLombschen) Kräfte nur über die Hülle wirken, d. h.

$$
F\left(k k^{\prime}\right)=D\left(k k^{\prime}\right)=0 .
$$

Dann gilt offensichtlich [vgl. (II, 16c, d) ]

$$
C_{\mathrm{N}}\left(k k^{\prime}\right)=T\left(k k^{\prime}\right),
$$

insbesondere also

$$
C_{\mathrm{N}}^{(0)}(11)=R_{0} I=T^{(0)}(11)=T_{0} I
$$

und $C_{\mathrm{N}}^{(1)}(12)=-i a r q^{(1)}=T^{(1)}(12)=-i a t q^{(1)}$, (II, 30b)

und somit (II, 29a). Die obige Voraussetzung läßt 
sich auch wellenmechanisch fundieren. Sie wurde insbesondere auch den Berechnungen der Gitterschwingungen in $\mathrm{NaJ}^{17}$ zugrunde gelegt. - Da wir in der Auswertung nur die Wechselwirkung mit nächsten Nachbarn berücksichtigen, ist überdies

$$
R_{0}=T_{0}=4 B\left(=4, \alpha_{1}\right) \text { und } \quad r=t=C\left(=\beta_{1}\right)
$$

zu setzen.

Diskussion der Formel (II, 29)

Die Formel (II, 29) unterscheidet sich von (II, 14) (abgesehen von den bei der numerischen Berechnung nicht so entscheidenden Änderungen im Nenner) vor allem im Zähler durch die zusätzlichen (dimensionslosen) Faktoren

$f_{1}=1-\frac{r}{p} \frac{e}{Y}, \quad f_{2}=1-\frac{e}{Y}$ bzw. $f_{3}=1-\frac{\alpha}{e} \frac{R_{0}}{Y}$

in den zwei Summanden. Eine Verkleinerung des berechneten (absoluten) Wertes von $e_{14}$ und damit eine bessere Annäherung an den experimentellen Wert ist also insbesondere dann zu erwarten, wenn diese Faktoren wesentlich kleiner als 1 sind.

\begin{tabular}{|c|c|c|c|c|}
\hline $\begin{array}{c}e \\
\text { in Elementar- } \\
\text { ladungen } \\
R_{0} \\
\text { in } 10^{3} \mathrm{dyn} / \mathrm{cm}\end{array}$ & $\begin{array}{c}Y \\
\text { in } \\
\text { Ele- } \\
\text { men- } \\
\text { tarl. }\end{array}$ & $f_{1} f_{2}$ & $f_{3}$ & $\begin{array}{c}e_{14} \\
\text { in } \\
10^{4} \mathrm{el} . \\
\text { stat. } \\
\mathrm{cgs} / \mathrm{cm}^{2}\end{array}$ \\
\hline $\begin{array}{c}e=0,96 \\
R_{0}=4 B=228,2\end{array}$ & $\begin{array}{l}4 \\
5 \\
7\end{array}$ & $\begin{array}{l}0,013 \\
0,172 \\
0,378\end{array}$ & $\begin{array}{r}-0,396 \\
-0,117 \\
0,202\end{array}$ & $\begin{array}{l}-18,0 \\
-15,2 \\
-15,5\end{array}$ \\
\hline $\begin{array}{c}e=1,5 \\
R_{0}=4 B=220,5\end{array}$ & $\begin{array}{l}4 \\
5 \\
7\end{array}$ & $\begin{array}{l}0,165 \\
0,288 \\
0,456\end{array}$ & $\begin{array}{l}0,137 \\
0,309 \\
0,507\end{array}$ & $\begin{array}{l}-12,9 \\
-14,9 \\
-26,1\end{array}$ \\
\hline $\begin{array}{c}e=1,5 \\
R_{0}=4 B=180\end{array}$ & $\begin{array}{l}4 \\
4,5 \\
5 \\
7\end{array}$ & $\begin{array}{l}0,165 \\
0,231 \\
0,288 \\
0,456\end{array}$ & $\begin{array}{l}0,295 \\
0,373 \\
0,446 \\
0,597\end{array}$ & $\begin{array}{l}-1,7 \\
-4,5 \\
-7,4 \\
-18,1\end{array}$ \\
\hline $\begin{array}{c}e=1,8 \\
R_{0}=4 B=180\end{array}$ & $\begin{array}{l}4 \\
4,1 \\
5 \\
7\end{array}$ & $\begin{array}{l}0,177 \\
0,190 \\
0,293 \\
0,455\end{array}$ & $\begin{array}{l}0,413 \\
0,427 \\
0,530 \\
0,664\end{array}$ & $\begin{array}{r}-2,8 \\
-4,0 \\
-15,2 \\
-40,5\end{array}$ \\
\hline
\end{tabular}

Tab. 3. Berechnung der piezoelektrischen Konstante $e_{14}$ für $\mathrm{ZnS}$ [nach Gl. (II, 29)].

Die Berechnung nach (II, 29) ist in Tab. 3 für verschiedene Hüllenladungen $Y$ des Schwefelions durchgeführt. In der ersten Oberzeile $(e=0,96$, $R_{0}=228,2$ ) sind die Werte der Tab. 2 (elastische Konstanten nach Bhagavantam) benutzt. Die piezo-

23 Die langen optischen Schwingungen brauchen hier nicht näher behandelt zu werden, da die für sie in ${ }^{17}$ erhaltenen Ergebnisse im Gegensatz zu den langen akustischen Schwingungen auch für das Zinkblendegitter gelten. elektrische Konstante wird zwar im Vergleich zur Berechnung nach Gl. (II, 14) beträchtlich erniedrigt, aber die Diskrepanzen gegenüber dem experimentellen Wert betragen immer noch einen Faktor von etwa 3 bis 4 . Nun zeigt sich aber gerade bei Anwendung des Hüllen-Modells auf die optischen Gitterschwingungen ${ }^{23}$ (vgl. Cochran ${ }^{17}$, S. 987 oben), daß die zur Bestimmung der effektiven Ionenladung $e=e^{*}$ benutzte Formel von Szigeti bzw. die äquivalenten Formeln zu modifizieren sind, und zwar ergibt sich als Zusammenhang zwischen der effektiven Ladung $e^{*}$ nach SzIGETr und der Ionenladung $e$ nach dem Hüllen-Modell [vgl. Anm. ${ }^{17}$, Gl. $(2,3,6)$ und Gl. $(2,3,7)]$

$$
e^{*}=e-d .
$$

Dabei ist $d$ die Abkürzung für $d \equiv Y \frac{T_{0}}{k_{2}+T_{0}}$ [vgl. ${ }^{17}$ und Anhang 3, Gl. $\left.\left(A_{3}, 7\right)\right]$. Nach den im Anhang 3 (Tab. 4) durchgeführten numerischen Abschätzungen ergibt sich $e=1,95$ bzw. $e=1,63$ Elementarladungen, je nach den verwendeten elastischen Konstanten. Diese Ladungswerte, die denen für das reine Ionenmodell $(e=2)$ schon sehr nahe kommen, scheinen zwar nach der vorherrschenden Auffassung über den Bindungscharakter von $\mathrm{ZnS}$ zu hoch, jedoch gibt es gerade einige neuere Experimente, nach denen $\mathrm{ZnS}$ nahezu als ein Ionenkristall aufzufassen ist und die folglich diese relativ hohen Ladungswerte stützen würden. Hierauf wird im Anhang 3 noch etwas näher eingegangen.

Für die Berechnung von $e_{14}$ benutzen wir abgerundete Werte, nämlich $e=1,5$ bzw. $e=1,8$. Die mit $e=1,5$ unter Beibehaltung der anderen Eingangswerte ${ }^{24}$ der Tab. 2 durchgeführte Berechnung zeigt die zweite Oberzeile in Tab. 3. In diesem Fall erniedrigt sich gegenüber der ersten Zeile der Zähler zwar noch erheblich, aber diese Verkleinerung wird durch den ebenfalls verkleinerten Nenner und den erhöhten Vorfaktor $4 e / a^{2}$ fast wieder kompensiert.

Es zeigt sich jetzt aber, daß schon eine relativ kleine Änderung insbesondere von $B$ zu einem wesentlich besseren Ergebnis führt. Benutzen wir nämlich statt der elastischen Konstanten $c_{11}$ nach BHAGAvantam die nach Voigt, so ist nach Anhang 3 $R_{0}=4 B=188,8 \cdot 10^{3} \mathrm{dyn} / \mathrm{cm}$. Für die Berechnung in der dritten Oberzeile von Tab. 4 ist der nach un-

24 Natürlich ändern sich auch alle Parameter, wie z. B. $B$ und $C$, in deren Bestimmungsgleichung [vgl. Gl. (II, 15a)] die Ladung eingeht. 
ten abgerundete Wert $R_{0}=180 \cdot 10^{3} \mathrm{dyn} / \mathrm{cm}$ benutzt, um insbesondere die Empfindlichkeit des Wertes für $e_{14}$ gegenüber der Wahl von $B$ noch deutlicher hervorzuheben. Die Werte in der vierten Oberzeile wurden mit $e=1,8$ berechnet unter Beibehaltung der sonstigen Eingangswerte der dritten Oberzeile.

Der experimentelle Wert

$$
e=-4,2 \cdot 10^{4} \mathrm{~cm}^{-1 / 2} \mathrm{~g}^{1 / 2} \sec ^{-1}
$$

wird, wie die dritte bzw. vierte Oberzeile zeigt, jetzt ungefähr für die Hüllenladungen $Y=4,5$ bzw. 4,1 $\cdot\left[4,803 \cdot 10^{-10}\right.$ el.stat.cgs $]$ angenommen. Mit $e=1,8$ wird der experimentelle Wert ohne die Erniedrigung von $R_{0}$ ebenfalls erreicht, jedoch erst für ungefähr $Y=3$.

Wie im Anhang 3 [Gl. $\left.\left(\mathrm{A}_{3}, 10\right)\right]$ gezeigt ist, ergeben sich auch aus dem Hüllen-Modell selbst Werte gleicher Größenordnung, nämlich $Y=5,03$ bzw. $Y=6,68$, je nach den verwendeten elastischen Konstanten.

Die oben erhaltenen Werte scheinen auch ganz vernünftig, wenn man z. B. mit den Untersuchungen von HAvinga $^{25}$ bei den Alkalihalogeniden vergleicht, nach denen die bei der Polarisation wirksamen Hüllenladungen für die meisten der Kristalle zwischen $Y=3$ und $Y=4$ liegen. Auch für die Hüllenladungen des J-Atoms in ${ }^{17}$ ergab sich nur ein Wert von 2,76 Elementarladungen. Daß die Hüllenladungen nicht ungefähr gleich der Gesamtladung aller Valenzelektronen, also ungefähr gleich 8 Elementarladungen sind, sondern wesentlich niedriger ausfallen, rührt nach Havinga vermutlich daher, daß von den Valenzelektronen hauptsächlich nur die äußeren pElektronen zur Polarisation beitragen.

Schließlich muß aber auch betont werden, daß bei der großen Unsicherheit in der Bestimmung der einzelnen Parameter und besonders der großen Empfindlichkeit des Endergebnisses gegen kleine Änderungen der Parameter im Augenblick noch nicht mit genügender Sicherheit entschieden werden kann, ob die Formel (II, 29) oder auch die vollständigeren Formeln im Anhang 1 wirklich zu einer quantitativ befriedigenden Bestimmung der piezoelektrischen Konstanten ausreichen oder ob die Übereinstimmungen in Tab. 3 mehr zufällig sind. Es ist aber jedenfalls mit einiger Sicherheit anzunehmen, daß die nach dem Hüllen-Modell beschriebene Polarisation der schweren Gitterbausteine einen ganz wesentlichen Einfluß auf den Piezoeffekt hat.

25 E. E. Havinga, Phys. Rev. 119, 1193 [1960].
$\mathrm{Da}$ auch für $c_{44}$ die allgemeinen Beziehungen durch Gl. (I, 11) in Teil I bereits gegeben sind, könnte man ohne große Schwierigkeiten auch die Beziehung für $c_{44}$ in beiden Modellen leicht explizit angeben. Wegen der $\mathrm{zu}$ erwartenden längeren Gleichungen und der Ungenauigkeit der Eingangsdaten sei jedoch an dieser Stelle davon abgesehen.

Zur Prüfung des Modells wäre es im übrigen sehr erfolgversprechend, wenn es gelänge, die bei den Schwingungen entstehenden inneren Verrückungen $\boldsymbol{u}^{(1)}=\boldsymbol{u}^{(1)}(2)-\boldsymbol{u}^{(1)}(1)$ und die Dipolmomente $\boldsymbol{m}^{(1)}$ (1) und $\boldsymbol{m}^{(1)}(2)$ und damit die Koeffizienten $u^{1}, U$ bzw. $v_{1}{ }^{1}, V_{1}$ und $v_{2}{ }^{1}, V_{2}$ experimentell zu bestimmen. Letztere dürften nämlich nach den Formeln nicht so empfindlich von der Genauigkeit der Parameterwerte abhängen. Darüber hinaus könnten sie, Gültigkeit des Modells vorausgesetzt, zur Festlegung weiterer, bisher wegen der zu geringen $Z$ ahl verfügbarer Meßdaten nicht bestimmbarer Parameter dienen. Von Birman ${ }^{26}$ wurde bereits die Möglichkeit diskutiert, die inneren Verrückungen mit den Methoden der RöntGen-Strahl-Beugung zu bestimmen.

Um mehr Vergleichsdaten zur Verfügung zu haben, wäre es auch wünschenswert, wenn die piezoelektrischen Konstanten weiterer Kristalle mit Zinkblendestruktur gemessen würden, insbesondere auch die von I - VII- und III - V-Verbindungen.

\section{Anhang}

\section{Gestalt der Koeffizienten unter Berücksichtigung der Polarisierbarkeit beider Atome}

Schreibt man das effektive Feld wieder in der Form (II, 3), so lauten die Gln. (II, 21), die den Zusammenhang zwischen den Verrückungen und den (Deformations-) Dipolmomenten bestimmen:

$$
\begin{aligned}
\boldsymbol{m}^{(1)}(1)= & \alpha_{1} \boldsymbol{K}^{(1)}+\alpha_{1} Q_{0}\left(\boldsymbol{m}^{(1)}(1)+\boldsymbol{m}^{(1)}(2)\right) \\
& -\frac{\alpha_{1}}{y_{1}} T^{(1)}(12) \cdot \boldsymbol{u}^{(0)}\left(\mathrm{A}_{1}, 1 \mathrm{a}\right) \\
& +\frac{\alpha_{1}}{y_{1}} T_{0} \boldsymbol{u}^{(1)}+\frac{\alpha_{1}}{y_{1} y_{2}} S_{\mathbf{0}} \boldsymbol{m}^{(1)}(2), \\
\boldsymbol{m}^{(1)}(2)= & \alpha_{2} \boldsymbol{K}^{(1)}+\alpha_{2} Q_{0}\left(\boldsymbol{m}^{(1)}(1)+\boldsymbol{m}^{(1)}(2)\right) \\
& -\frac{\alpha_{2}}{y_{2}} T^{(1)}(21) \cdot \boldsymbol{u}^{(0)}\left(\mathrm{A}_{1}, 1 \mathrm{~b}\right) \\
& -\frac{\alpha_{2}}{y_{2}} T_{0} \boldsymbol{u}^{(1)}+\frac{\alpha_{2}}{y_{1} y_{2}} S_{\mathbf{0}} \boldsymbol{m}^{(1)}(1) .
\end{aligned}
$$

[Die Koeffizienten erster Ordnung, also z. B.

$$
\alpha_{1} T^{(1)}(12) / y_{1} \quad \text { usw., }
$$

26 Siehe Anm. ${ }^{8}$ in Teil I. 
werden hier nicht benötigt, da sie in die zum Vergleich benutzte Gl. (I, 13) nicht eingehen. Da sie aber andererseits für die Vergleiche im Anhang 2 wesentlich sind, seien sie hier trotzdem explizit mit aufgeführt.]

Wenn man die Dipolmomente auf der linken Seite zusammenfaßt, ergibt sich

$$
\begin{aligned}
a_{11} \boldsymbol{m}^{(1)}(1)+a_{12} \boldsymbol{m}^{(1)}(2) & \left(\mathrm{A}_{1}, 2\right) \\
= & a_{1}\left[\boldsymbol{K}^{(1)}+\frac{1}{y_{1}}\left(T^{(1)}(21) \cdot \boldsymbol{u}^{(0)}+T_{0} \boldsymbol{u}^{(1)}\right)\right],
\end{aligned}
$$

$a_{21} \boldsymbol{m}^{(1)}(1)+a_{22} \boldsymbol{m}^{(1)}(2)$

Dabei ist

$$
=\alpha_{2}\left[\boldsymbol{K}^{(1)}-\frac{1}{y_{2}}\left(T^{(1)}(21) \cdot \boldsymbol{u}^{(0)}+T_{0} \boldsymbol{u}^{(1)}\right)\right] .
$$

$$
A=\left(\begin{array}{ll}
a_{11} & a_{12} \\
a_{21} & a_{22}
\end{array}\right)=\left(\begin{array}{cc}
1-\alpha_{1} Q_{0} & -\alpha_{1}\left(Q_{0}+S^{\prime}\right) \\
-\alpha_{2}\left(Q_{0}+S^{\prime}\right) & 1-\alpha_{2} Q_{0}
\end{array}\right)\left(\mathrm{A}_{1}, 2 \mathrm{a}\right)
$$

mit der Determinante

$$
D=|A|=1-\left(\alpha_{1}+\alpha_{2}\right) Q_{0}-\alpha_{1} \alpha_{2} S^{\prime}\left(2 Q_{0}+S^{\prime}\right),
$$

wobei $S^{\prime} \equiv S_{0} / y_{1} y_{2}$.

Indem man $\left(A_{1}, 2\right)$ von links mit der reziproken Matrix

$$
A^{-1}=\frac{1}{D}\left(\begin{array}{cc}
1-\alpha_{2} Q_{0} & \alpha_{1}\left(Q_{0}+S^{\prime}\right) \\
\alpha_{2}\left(Q_{0}+S^{\prime}\right) & 1-\alpha_{1} Q_{0}
\end{array}\right) \quad\left(A_{1}, 2 \mathrm{c}\right)
$$

multipliziert, erhält man $\boldsymbol{m}^{(1)}(1)$ und $\boldsymbol{m}^{(1)}(2)$ :

$$
\begin{gathered}
\boldsymbol{m}^{(1)}(1)=\frac{\alpha_{1}}{D}\left\{\left(1+\alpha_{2} S^{\prime}\right) \boldsymbol{K}^{(1)}\right. \\
-\left(\left[\frac{1}{y_{1}}+\frac{1}{y_{2}}\right] \alpha_{2} Q_{0}-\frac{1}{y_{1}}+\frac{a_{2}}{y_{2}} S^{\prime}\right) \\
\left.\quad \cdot\left(T(21) \cdot \boldsymbol{u}^{(0)}+T_{0} \boldsymbol{u}^{(1)}\right)\right\},
\end{gathered}
$$$$
\boldsymbol{m}^{(1)}(2)=\frac{\alpha_{2}}{D}\left\{\left(1+\alpha_{1} S^{\prime}\right) \boldsymbol{K}^{(1)}\right.
$$

$$
\begin{aligned}
+ & \left(\left[\frac{1}{y_{1}}+\frac{1}{y_{2}}\right] \alpha_{1} Q_{0}+\frac{1}{y_{2}}-\frac{a_{1}}{y_{1}} S^{\prime}\right) \\
& \left.\cdot\left(T(21) \cdot \boldsymbol{u}^{(0)}+T_{0} \boldsymbol{u}^{(1)}\right)\right\} .
\end{aligned}
$$

Indem man sich wieder $\boldsymbol{K}^{(1)}$ nach Gl. (II, 3) eingesetzt denkt, liest man durch Vergleich mit $(\mathrm{I}, 4)$ in Teil I ab:

$$
\begin{aligned}
& K_{1}=\frac{\alpha_{1}\left(1+\alpha_{2} S^{\prime}\right)}{D}, \quad K_{2}=\frac{\alpha_{2}\left(1+\alpha_{1} S^{\prime}\right)}{D}, \quad\left(\mathrm{~A}_{1}, 4\right) \\
& L_{1}=\frac{\alpha_{1}}{D}[ \quad\left(1+\alpha_{2} S^{\prime}\right) e Q_{0} \\
&\left.\quad-\left(\left(\frac{1}{y_{1}}+\frac{1}{y_{2}}\right) \alpha_{2} Q_{0}-\frac{1}{y_{1}}+\frac{\alpha_{2}}{y_{2}} S^{\prime}\right) T_{0}\right], \\
& L_{2}=\frac{a_{2}}{D}[\left(1+\alpha_{1} S^{\prime}\right) e Q_{0} \\
&\left.\quad+\left(\left(\frac{1}{y_{1}}+\frac{1}{y_{2}}\right) \alpha_{1} Q_{0}+\frac{1}{y_{2}}-\frac{\alpha_{1}}{y_{1}} S^{\prime}\right) T_{0}\right] .
\end{aligned}
$$

Die Koeffizienten $M_{1}, M_{2}, m_{1}{ }^{1}, m_{2}{ }^{1}$ ergeben sich schließlich wieder aus dem Vergleich von (II, 19) mit (I, $\left.I^{\prime} b\right)$ bzw. (I, 3) in Teil I $\left[M_{2}\right.$ und $m_{2}{ }^{1}$ sind wie durch (II, 24a) bzw. (II, 24c) gegeben]:

$M_{1}=\frac{T_{0}}{y_{1}}-e Q_{0}, \quad M_{2}=-\left(\frac{T_{0}}{y_{2}}+e Q_{0}\right), \quad\left(\mathrm{A}_{1}, 6 \mathrm{a}\right)$ $M_{1}{ }^{(1)} \equiv M^{(1)}(21)=i m_{1}{ }^{1} q^{(1)}=\frac{T^{(1)}(21)}{y_{1}}+e Q^{(1)}(21)$, d. h. mit (II, 8b) und (II, 20c) folgt

$$
m_{1}{ }^{1}=a\left(\frac{t}{y_{1}}-\frac{p}{e}\right)
$$

und wie in (II, 24c) :

$$
m_{2}{ }^{1}=-a\left(\frac{t}{y_{2}}+\frac{p}{e}\right) .
$$

$C$ und $c^{1}$ sind als von der Polarisation unabhängige Koeffizienten wieder durch Gl. (II, 11) bzw. (II, 8e) gegeben.

Berücksichtigt man wie früher nur die Wechselwirkung mit nächsten Nachbaratomen und setzt voraus, daß die nicht-Coulombschen Kräfte allein über die Hülle übertragen werden, so ist wie früher $R_{0}=T_{0}=4 B\left(=4 \alpha_{1}\right)$ und $r=t=C\left(=\beta_{1}\right)$ zu setzen.

Auf das Einsetzen in die Endformel für die piezoelektrische Konstante [Gl. (I, 13)] sei jedoch hier verzichtet, da diese dann sehr lang und unübersichtlich würde.

\section{Beweis der Identität der Gln. $(I, 9)$ und $(I, 13)$}

Zunächst bestätigt man leicht, daß für alle betrachteten Modelle die drei Beziehungen erfüllt sind:

$$
\begin{aligned}
L_{1}+L_{2} & =M_{1} K_{1}+M_{2} K_{2}, & & \left(\mathrm{~A}_{2}, l \mathrm{a}\right) \\
l_{1}{ }^{1}+l_{2}{ }^{1} & =K_{1} m_{1}{ }^{1}+K_{2} m_{2}{ }^{1}, & & \left(\mathrm{~A}_{2}, 1 \mathrm{~b}\right) \\
M_{1} l_{1}{ }^{1}+M_{2} l_{2}{ }^{1} & =L_{1} m_{1}{ }^{1}+L_{2} m_{2}{ }^{1} . & & \left(\mathrm{A}_{2}, 1 \mathrm{c}\right)
\end{aligned}
$$

Für das allgemeine Hüllen-Modell sind dabei alle Ausdrücke aus Anhang 2 zu entnehmen, bis auf $l_{1}{ }^{1}$ und $l_{2}{ }^{1}$, die hier nachträglich angeführt seien [abzulesen aus $\left.\left(A_{1}, 3\right)\right]$ :

$$
\begin{aligned}
l_{1}{ }^{1}=-\frac{\alpha_{1} a}{D}\{ & \left(1+\alpha_{2} S^{\prime}\right) \frac{p}{e} \\
& \left.+\left[\left(\frac{1}{y_{1}}+\frac{1}{y_{2}}\right) \alpha_{2} Q_{0}-\frac{1}{y_{1}}+\frac{\alpha_{2}}{y_{2}} S^{\prime}\right] t\right\}, \\
l_{2}{ }^{1}=-\frac{\alpha_{2} a}{D}\{ & \left(1+\alpha_{1} S^{\prime}\right) \frac{p}{e} \\
& \left.-\left[\left(\frac{1}{y_{1}}+\frac{1}{y_{2}}\right) \alpha_{1} Q_{0}+\frac{1}{y_{2}}-\frac{a_{1}}{y_{1}} S^{\prime}\right] t\right\} .
\end{aligned}
$$

Nach Einsetzen von (I, 5a) und (I, 6c) ergibt sich als rechte Seite von Gl. (I, 9) (bis auf den Faktor $\left.1 / v_{a}\right)$ :

$$
\begin{aligned}
& v_{1}{ }^{1}+v_{2}{ }^{1}-e u^{1}=-\left(L_{1}+L_{2}-e\right) \frac{c^{1}+M_{1} l_{1}{ }^{1}+M_{2} l_{2}{ }^{1}}{C+M_{1} L_{1}+M_{2} L_{2}} \\
& +l_{1}{ }^{1}+l_{2}{ }^{1} \text {. } \\
& \left(\mathrm{A}_{2}, 3\right)
\end{aligned}
$$


Andererseits erhält die rechte Seite von Gl. (I, 13) nach Einsetzen von $(\mathrm{I}, 5 \mathrm{~b})$ und $(\mathrm{I}, 6 \mathrm{~d})$ die Gestalt (wieder bis auf den Faktor $1 / v_{a}$ ):

$$
\begin{array}{r}
U c^{1}+V_{1} m_{1}{ }^{1}+V_{2} m_{2}{ }^{1}=-\frac{M_{1} K_{1}+M_{2} K_{2}-e}{C+M_{1} L_{1}+M_{2} L_{2}}\left(\mathrm{~A}_{2}, 4\right) \\
\cdot\left(c^{1}+L_{1} m_{1}{ }^{1}+L_{2} m_{2}{ }^{1}\right)+K_{1} m_{1}{ }^{1}+K_{2} m_{2}{ }^{1} .
\end{array}
$$

Man sieht jetzt sofort, daß wegen $\left(A_{2}, 1\right)$ beide Ausdrücke übereinstimmen.

Zum Beweis der Gln. $\left(A_{2}, 1\right)$ wurden hier zwar die speziellen Modellgleichungen benutzt, die Gln. $\left(A_{2}, 1\right)$ gelten aber vermutlich allgemein, woraus dann auch die Identität von $\left(A_{2}, 3\right)$ und $\left(A_{2}, 4\right)$ und damit von (I,9) und $(\mathrm{I}, 13)$ im allgemeinen Fall folgen würde.

3. Bestimmung der „effektiven“ Ionenladung e und der Hüllenladung $Y$ unter zusätzlicher Benutzung der Formeln für die langen optischen Gitterschwingungen bei Annahme des Hüllen-Modells.

\section{Diskussion der Ergebnisse}

Nimmt man zur Berechnung der langen optischen Gitterschwingungen in Kristallen mit tetraedischer Symmetrie an, daß die Polarisation allein durch das von den Gitterschwingungen erzeugte elektrische Feld hervorgerufen wird, so erhält man nach Anm. ${ }^{2}$ als Frequenzen der langen optischen Gitterschwingungen $^{27}$ :

$$
M \omega_{\mathrm{tr}}{ }^{2}=M \omega_{0}^{2}=R_{0}-\frac{4 \pi e^{* 2}}{9 v_{a}}\left(\varepsilon_{\infty}+2\right), \quad\left(\mathrm{A}_{3}, 1\right)
$$

für die transversalen Schwingungen und

$$
M \omega_{1}^{2}=R_{0}+\frac{8 \pi e^{* 2}}{9 v_{a} \varepsilon_{\infty}}\left(\varepsilon_{\infty}+2\right)
$$

für die longitudinalen Schwingungen. $M$ ist dabei die reduzierte Masse, $\varepsilon_{\infty}=n^{2}$ die optische Dielektrizitätskonstante. Führt man in $\left(\mathrm{A}_{3}, 2\right) \omega_{1}^{2}=\omega_{\operatorname{tr}^{2}}{ }^{2} \varepsilon_{0} / \varepsilon_{\infty}$ (Lyddane-Sachs-Teller-Beziehung) ein, so kann man die beiden Gleichungen zur Bestimmung von $e^{*}$ und $R_{0}$ ausnutzen, da alle sonstigen Größen sich unmittelbar aus Experimenten entnehmen lassen. Subtrahiert man $\left(A_{3}, 1\right)$ von $\left(A_{3}, 2\right)$ und löst nach $e^{*}$ auf, so folgt

$$
e^{*}=\omega_{0}\left(M v_{a}\right)^{1 / 2}\left(\frac{\varepsilon_{0}-\varepsilon_{\infty}}{4 \pi}\right)^{1 / 2} \frac{3}{\varepsilon_{\infty}+2}
$$

[ $=$ Gl. $(9,31)$ in Anm. $\left.{ }^{2}\right]$.

Addiert man andererseits beide Gleichungen [nach Multiplikation von $\left(\mathrm{A}_{3}, 2\right)$ mit $\varepsilon_{\infty} / 2$ ] und löst nach

27 Zur Herleitung von $\left(\mathrm{A}_{3}, 1\right)$ setze man $b_{11}$ nach Anm. ${ }^{2}$, Gl. $(9,17)$, S. 106 in Gl. $(7,6)$, S. 84, ein, wobei man $a=\alpha_{1}+\alpha_{2}$ noch nach der Formel von Lorentz-Lorenz durch $\varepsilon_{\infty}$ und $\nu_{a}$ ausdrücke. Bei der Herleitung von Gl. $\left(\mathrm{A}_{3}, 2\right)$,
$R_{0}$ auf, so ergibt sich:

$$
R_{0}=M \omega_{0}^{2} \frac{\varepsilon_{0}+2}{\varepsilon_{\infty}+2}
$$

[ $=$ Gl. $(9,21)$ in Anm. ${ }^{2}$ ].

Aus Gl. $\left(\mathrm{A}_{3}, 3\right)$, die von Szigeti hergeleitet wurde, sind die effektiven Ladungen $e^{*}$ für eine ganze Reihe von Kristallen berechnet worden (s. z. B. Tab. 20 in Anm. ${ }^{2}$ ). Die erhaltenen teilweise erheblichen Abweichungen dieser Ladungen von den ganzzahligen Werten, die man für reine Ionenkristalle zu erwarten hätte, werden gewöhnlich als ein Maß für entsprechend große homöopolare Bindungsanteile angesehen. Dies ist indes nur dann berechtigt, wenn das zugrunde gelegte Modell insbesondere hinsichtlich der Entstehung der elektronischen Polarisation für die untersuchten Kristalle wirklich zutrifft.

Cochran ${ }^{17}$ u. a. haben nun aber gezeigt, daß sich unter der Annahme des Hüllen-Modells zwar äußerlich ähnliche Gleichungen ergeben, jedoch mit einigen wesentlichen Modifikationen. Man hat nämlich in $\left(A_{3}, 1\right)$ und $\left(A_{3}, 2\right)$ und folglich auch in $\left(A_{3}, 3\right)$ bzw. $\left(\mathrm{A}_{3}, 4\right) R_{0}$ durch

$$
R_{0}{ }^{\prime} \equiv R_{0}-\left(d^{2} / \alpha\right)
$$

zu ersetzen, und $e^{*}$ steht mit der Ladung $e$ nach dem Hüllen-Modell in dem Zusammenhang:

$$
e^{*}=e-d
$$

[vgl. Gl. $(2,3,6)$ und Gl. $(2,3,7)$ in Anm. ${ }^{17}$ ]. $e$ ist als die wahre Ladung der Ionen im ruhenden Kristall aufzufassen. $d$ ist eine Abkürzung für [vgl. Gl. $(2,3,3)$ ebendort]

$$
d \equiv T_{0} Y /\left(k_{2}+T_{0}\right)=R_{0} Y /\left(k_{2}+R_{0}\right), \quad\left(\mathrm{A}_{3}, 7\right)
$$

wobei die letztere Form nur in der benutzten Näherung gilt, daß die Kräfte nur über die Hülle wirken. Führt man nach Gl. (II, 20) noch die Polarisierbarkeit $\alpha$ ein, so folgt:

$$
d=\alpha R_{0} / Y .
$$

In den Formeln $\left(A_{3}, 5\right)$ bis $\left(A_{3}, 7\right)$ wurde dabei vorausgesetzt, daß die Polarisation des Kations vernachlässigbar ist. Die allgemeineren Gleichungen sind im Anhang 3 von Anm. ${ }^{17}$ zu finden. - Andererseits gilt bei Vernachlässigung der Kopplungsparameter zweiter und höherer Nachbarn die Beziehung [vgl. Gl. (II, 15a) ]

$$
R_{0}=4 B=4 a c_{11}-1,00 e^{2} / v_{a} .
$$

Setzt man dies in $\left(A_{3}, 5\right)$ ein, so lassen sich die

wozu man z. B. von der (unnumerierten) Gleichung unter Gl. $(8,20)$, S. 93 , ausgehe, setze man außerdem ein $b_{12}=b_{21}$ nach Gl. $(9,10)$, S. $105[=$ Gl. $(9,18)$, S. 106], und $1+4 \pi b_{22}=\varepsilon_{\infty}$ nach Gl. $(7,8)$, S. 84 . 
beiden Unbekannten $e$ und $d$ aus $R_{0}^{\prime}$ und $e^{*}$ bestimmen. Das Ergebnis zeigt Tab. 4.

\begin{tabular}{|c|c|c|}
\hline $\begin{array}{c}\text { Elastische } \\
\text { Konstanten } \\
\text { nach }\end{array}$ & $\begin{array}{c}e \\
\text { in } \\
\text { Elementarlad. }\end{array}$ & $\begin{array}{c}d \\
\text { in } \\
\text { Elementarlad. }\end{array}$ \\
\hline $\begin{array}{l}\text { BHAGAVANTAM } 11 \\
\text { VoIGT } 12\end{array}$ & $\begin{array}{c}1,95 \\
(-0,26) \\
1,63 \\
(0,06)\end{array}$ & $\begin{array}{c}0,99 \\
(-1,22) \\
0,67 \\
(-0,90)\end{array}$ \\
\hline
\end{tabular}

Tab. 4.

Dabei sind die Werte der (sicherlich unzutreffenden) zweiten Lösung der quadratischen Gleichung für $e$ und $d$ nur zum Vergleich (in Klammern) mit aufgeführt.

Unsicherheiten in der Bestimmung von $e$ und $d$ sind, Gültigkeit des Hüllen-Modells vorausgesetzt, neben der Unsicherheit in den Werten für die elastischen Konstanten im wesentlichen nur dadurch gegeben, daß der Einfluß der Kopplungsparameter zweiter (und höherer) Nachbarn in $\left(\mathrm{A}_{3}, 8\right)$ nicht berücksichtigt ist und man nicht genau weiß, wie groß der Anteil der Kräfte kurzer Reichweite ist, die nicht über die Hüllen wirken.

Die nach Tab. 4 erhaltenen Werte für die Ionenladungen scheinen, wie bereits auf S. 223 bemerkt, nach der vorherrschenden Auffassung über den Bindungscharakter von $\mathrm{ZnS}$ sehr groß, da sie für eine weitgehend heteropolare (ionogene) Bindung sprechen würden, während von vielen Autoren ein beträchtlicher homöopolarer Bindungsanteil angenommen wird. Wegen einer Zusammenstellung und

28 J. L. Birman, Phys. Rev. 109, 810 [1958].

29 A. Faessler u. M. Goemring, Z. Phys. 142, 558 [1955].

30 A. Faessler u. P. Mecke, Z. Elektrochem. 59, 425 [1955].
Diskussion diesbezüglicher Arbeiten sei z. B. auf BIRMAN ${ }^{28}$ hingewiesen. Neben einigen anderen Argumenten ist es, wie bereits erwähnt, insbesondere die effektive Ladung nach SzIgeri von $e \approx 1$, aus der auf einen größeren homöopolaren Bindungsanteil geschlossen wird. Wie aber oben bereits betont, ist diese Ladung allein bei Gültigkeit des Hüllenmodells kein eindeutiges $\mathrm{Maß}$ mehr für die wahre Ionenladung und nur noch eine Rechengröße. - Andererseits ergibt sich aus den K-Emissionsspektren, wie die eingehenden Untersuchungen von FAEssLeR und Mitarb. ${ }^{29-31}$ zeigen, daß $\mathrm{ZnS}$ nahezu als ein Ionenkristall aufzufassen ist. Auch die Berechnungen der Bindungsenergie in $\mathrm{ZnS}$ nach Asano und Tomishima ${ }^{32}$ führen auf eine Ladung von rund $e=1,7$.

Mit den Werten der Tab. 4 folgt nun nach $\left(\mathrm{A}_{3}, 8\right)$

$R_{0}=221,5 \cdot 10^{3} \mathrm{dyn} / \mathrm{cm}\left(\mathrm{c}_{11}\right.$ nach Anm. $\left.{ }^{11}\right)$, bzw.

$R_{0}=188,8 \cdot 10^{3} \mathrm{dyn} / \mathrm{cm}\left(c_{11}\right.$ nach Anm. $\left.{ }^{12}\right)$.

Hiermit läßt sich nach $\left(A_{3}, 7 \mathrm{a}\right)$ jetzt auch die Hüllenladung $Y$ berechnen, mit dem Ergebnis

$\begin{aligned} & Y=5,03 \text { Elementarladungen, } \quad\left(\mathrm{A}_{3}, 10\right) \\ & \text { bzw. } \quad Y=6,68 \text { Elementarladungen. }\end{aligned}$

Für die Förderung der Arbeit möchte ich Herrn Prof. E. Krautz herzlich danken, ebenfalls Herrn Prof. A. Faessler für seine sehr interessante Diskussion über den Bindungscharakter in $\mathrm{ZnS}$. Für die freundliche Durchführung eines Teils der numerischen Rechnungen schulde ich Frau U. Zollfrank und Herrn K. Lippert Dank.

31 A. Faessler, Proc. Int. Conf. on Semiconductor Physics, Prag 1960, S. 914.

32 S. Asano u. Y. Tomishima, J. Phys. Soc. Japan 11, 664 [1956], zitiert in Anm. ${ }^{28}$. 